\title{
Developing the Minimizing Re-Transmissions (MRT) Technique for Broadcasting in Wired Networks
}

\author{
Nguyen Xuan Tien ${ }^{1}$, Semog Kim² ${ }^{2}$ and Jong Myung Rhee \\ Department of Information and Communications Engineering - Myongji University - \\ Republic of Korea \\ E-mail: ${ }^{1}$ nxtien@gmail.com; ${ }^{2}$ kimsemog@empas.com; ${ }^{3} j m r 77 @ m j u . a c . k r$
}

\begin{abstract}
Broadcasting is the simplest way to send a message from one node to all the other nodes in a network. Simple flooding is the simplest form of broadcasting that provides important control, route discovery, and network information update functionality for unicast and multicast protocols. However, simple flooding generates too many broadcast message duplications in networks. The Minimizing Re-Transmissions (MRT) approach has been developed to reduce broadcast duplication in ad hoc wireless networks by minimizing the number of retransmitting nodes based on the network topology information. While MRT is one of the most efficient broadcasting techniques for ad hoc wireless networks, when MRT is applied for wired networks, it still generates broadcast duplication in the networks. In this paper, we develop a variant of MRT for wired networks called MRT for wired networks (wMRT) to completely remove broadcast duplication in wired networks. In addition to minimizing the number of retransmitting nodes, wMRT also minimizes the number of retransmitting ports of each node in wired networks. This results in no broadcast duplication in wired networks. The traffic performance of wMRT is analyzed, evaluated, and compared to that of the simple flooding and MRT techniques. Simulations are conducted using the OMNeT++ simulation tool in order to validate the traffic performance analysis. The analyzed and simulated results show that the wMRT completely removes broadcast duplication in wired networks, thus saving a significant amount of network bandwidth, as well as improving network traffic performance.
\end{abstract}

Keywords: minimizing retransmissions (MRT), MRT for wired networks, efficient broadcasting, efficient flooding

\section{Introduction}

Broadcasting is the process of sending a message from one node to every other node in a network. During broadcasting, the source node usually requires the assistance of its immediate neighbors to rebroadcast the message to their neighboring nodes. This process continues until all nodes in the network have received at least a copy of the message [1]. Broadcasting provides route establishment and control functionality for a number of unicast and multicast protocols. Many routing protocols, such as AODV [2], DSR [3], OSPF [4], and IS-IS [5], rely on broadcasting for failure notification, route discovery and maintenance, and network topology updating, or simply for sending control or maintenance messages. Broadcasting techniques are also used in Video-on-Demand (VOD) systems. Broadcasting protocols are proved to be efficient for transmitting most of the popular videos in VOD systems [6]. Additionally, broadcasting supports to maintain valuable global information of peer-to-peer (P2P) networks. In P2P networks,

* Corresponding Author 
broadcasting disseminates a message to all peers; in the reverse direction, it can aggregate the responses from peers [7].

The most basic form of broadcasting is "simple flooding" (SP). In this form, every node rebroadcasts a broadcast message when it first receives the message [8-11]. In other words, each node retransmits the broadcast message once. When a node receives a broadcast message, it determines if it has transmitted the message before; if not, the node rebroadcasts the message. This process allows a broadcast message to be delivered throughout the network. Simple flooding ends when all nodes in the network have received and retransmitted the broadcast message at least once. This simple scheme guarantees that a broadcast message can reach all nodes if the network is connected. The main problem with simple flooding, however, is that too many retransmitting nodes in a network generate too many broadcast message duplications. Generally, when a broadcast message is sent in an $n$-node network, a total of n- 1 retransmitting nodes rebroadcast the message once. The large number of retransmitting nodes causes significant broadcast message duplication. High broadcast message duplication can result in high bandwidth consumption in networks.

Several broadcasting techniques have been developed and proposed in order to reduce the number of retransmissions while attempting to ensure that a broadcast message is delivered to each node in the network. Many broadcasting methods have been proposed for mobile ad hoc networks (MANETs). The broadcasting methods can be broadly classified into the following categories: heuristic-based, area-based, neighbor cover-agebased, cluster-based, and tree-based broadcasting methods. Broadcasting methods for MANETs were reviewed and explored in [8-12]. Some efficient broadcasting protocols for VOD systems have been proposed. These broadcasting protocols can be subdivided in to three groups [6]. Protocols in the first group [14-15] are pyramid-based protocols that partition the video into increasing size of segments and transmit them in logical channels of the same bandwidth. Broadcasting protocols of the second group [16-18] called harmonic-based protocols divide the video into equal size segments and transmit them in logical channels of decreasing bandwidth. Protocols of the third group [19-20] are hybrid of pyramid-based protocols and harmonic-based protocols. They partition each video into fixed size segments and map them into a small number of data streams of equal bandwidth. Broadcasting techniques in P2P networks have been proposed in [21-23]. Broadcasting on a structured P2P network usually disseminates messages along the edges of a spanning tree [7]. Some broadcasting techniques [21-22] presented how a peer broadcast a message to peers within a range. These techniques implicitly construct a spanning tree in a top-down and on-demand fashion by selecting proper routing entries in their own routing tables. Another technique [23] proposed to build and maintain a spanning tree explicitly on a bottom-up fashion to support operations such as information aggregation. When a peer wants to broadcast a message, it first sends the message to the root of the spanning tree.

An effective broadcasting technique minimizes the number of nodes that are involved in re-broadcasting broadcast messages. Among the various broadcasting techniques, Minimizing Re-Transmissions (MRT) [12-13] is one of the most efficient. The MRT technique was developed to work with link state routing protocols in ad hoc wireless networks. MRT uses link state information obtained by the routing protocols to provide an efficient flooding technique in ad hoc wireless networks. The primary goal of MRT is to minimize the number of retransmitting nodes in a network; it thus significantly reduces broadcast message duplication in the network. The MRT technique works very efficiently when it is applied to ad hoc wireless networks. When MRT is used to send broadcast traffic in wired networks, however, it generates broadcast duplication in the networks.

In this paper, we develop a variant of MRT for wired networks called wMRT. The key idea of wMRT is to minimize not only the number of retransmitting nodes, but also the number of retransmitting nodes' ports in wired networks. Like MRT, the wMRT 
technique allows a minimum number of nodes in a wired network to retransmit broadcast messages. The rest of the nodes are not allowed to retransmit the broadcast messages. The wMRT approach also allows a minimum number of nodes' ports in the wired network to retransmit broadcast messages. By minimizing both the number of retransmitting nodes and the number of retransmitting ports, wMRT completely removes broadcast duplication in wired networks.

The remainder of this paper is organized as follows. In Section 2, we introduce the MRT technique. In Section 3, we present the proposed wMRT. Then, in Section 4, we analyze, evaluate, and compare the traffic performance of wMRT to that of both simple flooding and MRT. In Section 5, we present various simulations, and we describe their results in order to evaluate and validate the traffic performance analysis of wMRT. Finally, we provide our conclusions and suggestions for future work in Section 6.

\section{The MRT Technique}

The purpose of the MRT technique [12] is to minimize the number of retransmitting nodes in a network. Based on broadcast roles of nodes in the network, the MRT technique classifies them into two types of nodes: active-broadcast nodes and passive-broadcast nodes.

- Active-broadcast nodes: Active-broadcast nodes are nodes in a network that are allowed to retransmit a broadcast message when it receives the message for the first time in the network.

- Passive-broadcast nodes: Passive-broadcast nodes are nodes in a network that are not allowed to retransmit broadcast messages in the network.

When receiving a broadcast message, the active-broadcast nodes retransmit the message that is received for the first time, whereas the passive-broadcast nodes do not rebroadcast the message.

In order to classify and assign broadcast roles to nodes in a network, the MRT technique establishes and maintains a broadcast tree of the network; based on this broadcast tree, MRT assigns broadcast roles to network nodes. The processes of establishing the broadcast tree and assigning broadcast roles to nodes are described as follows.

\subsection{Establishing the Broadcast Tree}

Only one broadcast tree is established for the network in connected networks. The broadcast tree consists of one root, intermediate nodes and leaves. The MRT algorithm for establishing the broadcast tree is described as follows [12].

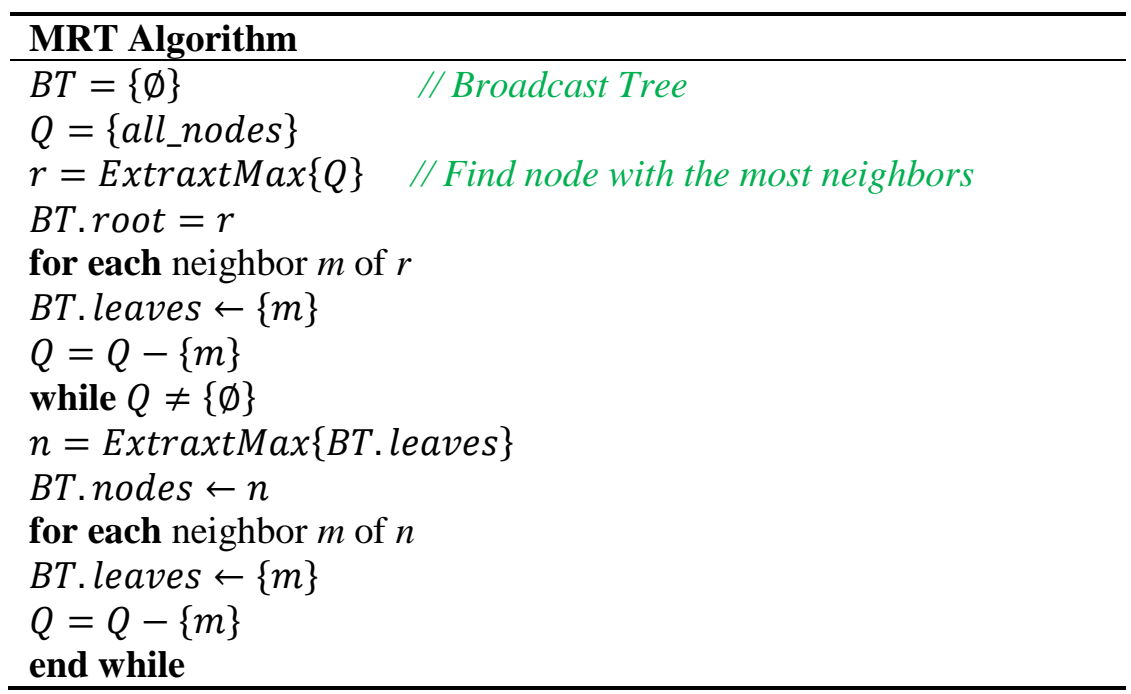


A sample network with 25 nodes is shown in Figure 1. Figure 2 shows the broadcast tree of the sample network that is constructed from the MRT technique.

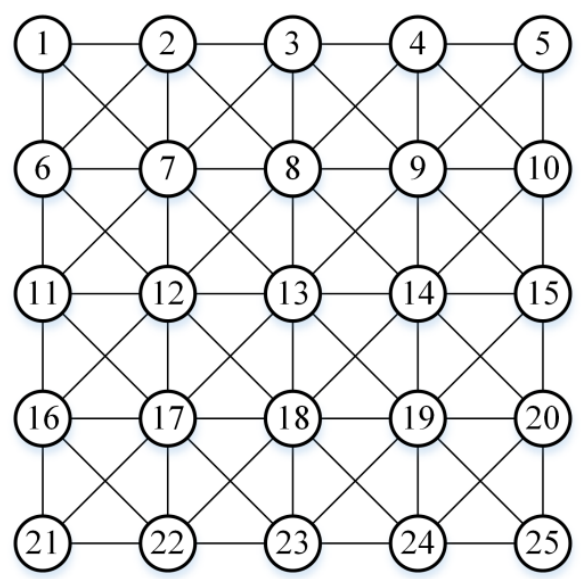

Figure 1. A Sample Network with 25 Nodes

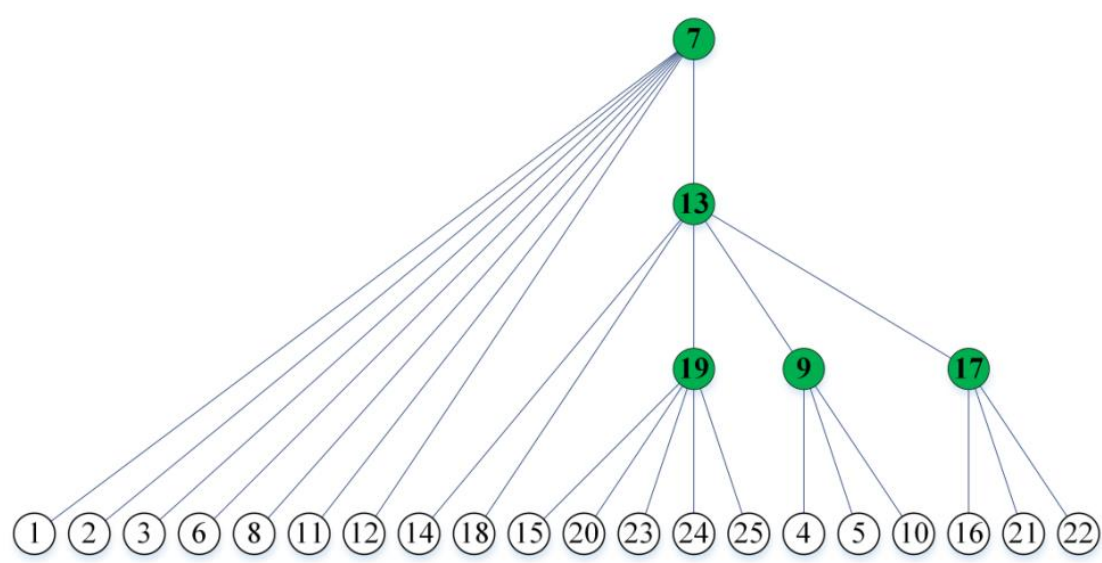

Figure 2. The Broadcast Tree of the Sample Network in Figure 1

\subsection{Assigning Broadcast Roles}

Once the broadcast tree is built, MRT assigns broadcast roles to nodes in the network. The root and the intermediate nodes of the broadcast tree are set to activebroadcast nodes, whereas the leaves are set to passive-broadcast nodes.

For the sample network in Figure 1, five active-broadcast nodes and twenty passive-broadcast nodes are assigned, based on the broadcast tree of the network, as shown in Figure 3.

\subsection{MRT Issues}

When a node sends a broadcast message, active-broadcast nodes will receive the broadcast message and flood it to all their ports, except the port in which the broadcast message is received. Passive-broadcast nodes will not retransmit the received message. Because all passive-broadcast nodes are connected to activebroadcast nodes, the broadcast message is delivered through the network.

In wired networks, a passive-broadcast node can connect to a few activebroadcast nodes. This causes broadcast duplications to be received at the passivebroadcast node. 


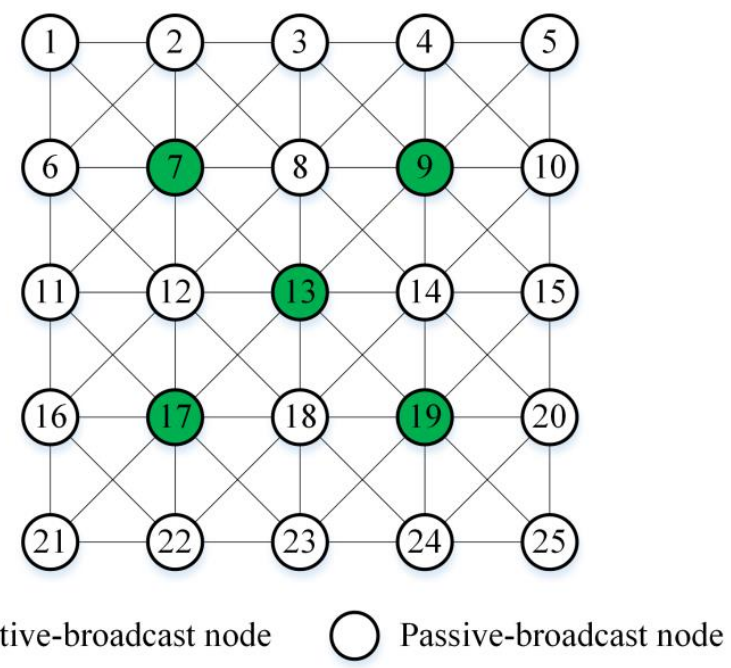

\section{Figure 3. Broadcast Roles of Nodes in the Sample Network in Figure 1}

For the sample network in Figure 3, when node 1 sends a broadcast message, it sends the broadcast message over all its ports. Active-broadcast node 7 receives and floods the broadcast message to all its ports, except the port connecting to node 1 , in which the broadcast message is received. Active-broadcast node 13 receives and floods the message to all its ports, except the port connecting to node 7. Similarly, the other active-broadcast nodes 9,17 , and 19 receive and flood the message to all of their ports, except the port connecting to node 13. In that case, passive-broadcast node 8 will receive three copies of the broadcast messages that are retransmitted by active-broadcast nodes 7,9 , and 13 . The same situation is true for passive-broadcast nodes 14 and 18. In other words, MRT generates broadcast duplication in wired networks.

\section{The Proposed wMRT Technique}

The proposed wMRT is a variant of MRT that was developed for efficiently broadcasting in wired networks. Like MRT, the wMRT approach works with link state protocols and uses link state information obtained by the routing protocols.

The primary goal of wMRT is to minimize not only the number of retransmitting nodes, but also the number of retransmitting ports of nodes in a wired network. Unlike MRT, which allows active-broadcast nodes to flood received broadcast messages to all of their ports, the wMRT approach allows the active-broadcast nodes to exclusively send the broadcast messages over their active-broadcast ports; wMRT thus completely removes broadcast message duplication in the wired network.

\subsection{Definitions}

As a variant of MRT, the wMRT technique uses all of the terms defined in MRT [12]. Along with the terms defined in MRT, wMRT defines a few of its own new terms.

In order to remove broadcast duplication in wired networks, the wMRT technique defines two types of node ports: active-broadcast ports and passive-broadcast ports.

Definition 1 (Active-broadcast port): An active-broadcast port is a port of a node that is used to retransmit broadcast messages.

Definition 2 (Passive-broadcast port): A passive-broadcast port is a port of a node that is not used to retransmit broadcast messages.

Each passive-broadcast node has only one active-broadcast port, whereas each activebroadcast node can have some active-broadcast ports. The port type is set based on the 
type of the link that the port is connected to. The wMRT approach also defines two types of links in a wired network: active-broadcast links and passive-broadcast links.

Definition 3 (Active-broadcast link): An active-broadcast link is a link in a wired network that is used to deliver broadcast messages in the wired network.

Definition 4 (Passive-broadcast port): A passive-broadcast link is a link in a wired network that is not used to deliver broadcast messages in the wired network.

\subsection{The wMRT Operations}

The proposed wMRT consists of three stages, as follows:

1) Building the broadcast tree of a network;

2) Assigning broadcast roles to nodes in the network;

3) Assigning broadcast roles to node ports in the network.

The first two stages of wMRT are similar to those of MRT [12]. The final stage is used to minimize the number of active-broadcast ports in the network. This stage is the main enhancement of the proposed wMRT compared with MRT for wired networks.

In the MRT technique, when an active-broadcast node receives a broadcast message, it floods the broadcast message to all of its ports, except for the port that receives the message. The drawback of this approach is that it causes broadcast duplications to be received at the passive-broadcast nodes. In order to remove the broadcast duplication from the passive-broadcast nodes as well as from the whole network, wMRT allows active-broadcast nodes to send received broadcast messages exclusively over their activebroadcast ports, instead of flooding all of their ports.

The process of assigning broadcast roles to nodes' ports consists of the following two steps: (1) assigning broadcast roles to links and (2) assigning broadcast roles to ports.

3.2.1. Assigning Broadcast Roles to Links: For the MRT technique, all links connected to active-broadcast nodes are active-broadcast links. The active-broadcast links of the sample network in Figure 1 under the MRT technique are shown in Figure 4.

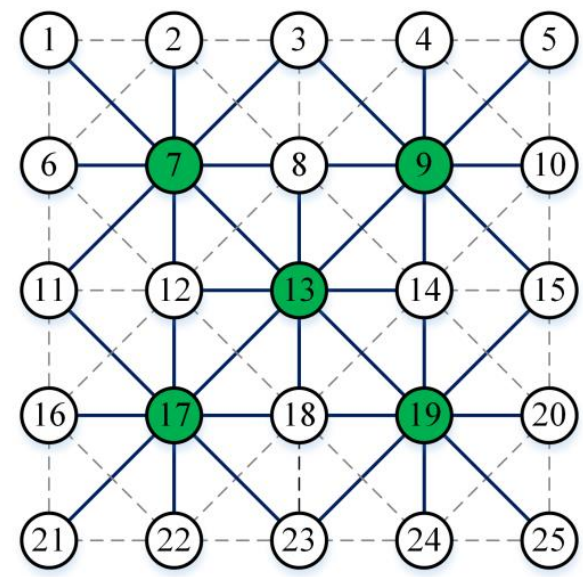

— Active-broadcast link

Passive-broadcast link

\section{Figure 4. Active-Broadcast Links of the Sample Network under MRT}

The wMRT technique assigns broadcast roles to links in a wired network based on the broadcast tree of the network. Links that form the edges of the broadcast tree are set to active-broadcast links; the rest of the links are passive-broadcast links. The activebroadcast links of the sample network in Figure 1 are shown in Figure 5. 


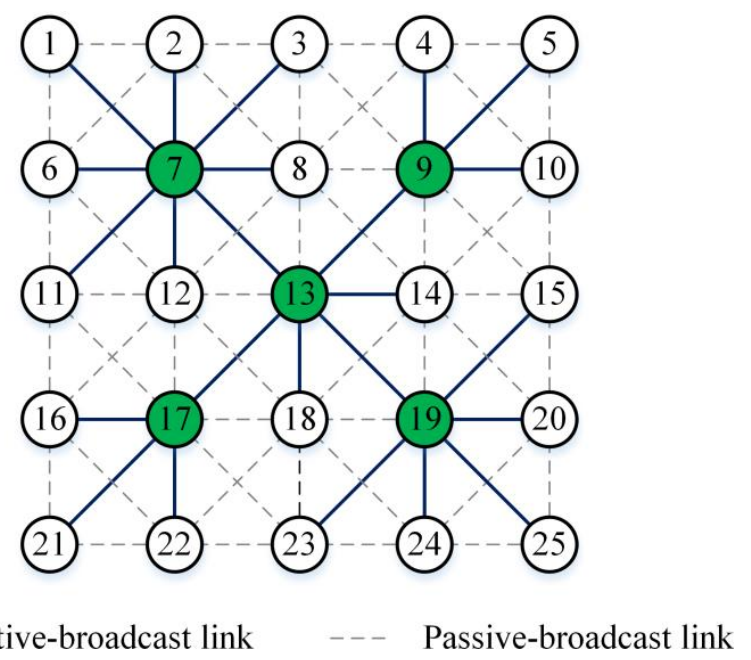

Figure 5. Active-Broadcast Links of the Sample Network under wMRT

Therefore, in the wMRT approach, each passive-broadcast node only has one activebroadcast link that connects to an active-broadcast node. For the same network, the number of active-broadcast links in the wMRT technique is much less than that in the MRT technique. Intuitively, there is no broadcast duplication at passive-broadcast nodes using the wMRT technique.

3.2.2. Assigning Broadcast Roles to Ports: Based on the active-broadcast links, each node in the wired network assigns broad-cast roles to its ports. Ports that are connected to active-broadcast links are set to active-broadcast ports, whereas ports that are connected to passive-broadcast links are set to passive-broadcast ports.

Under the wMRT approach, when an active-broadcast node receives a broadcast message, it exclusively sends the message over its active-broadcast ports.

The pseudocode of the wMRT algorithm is described as follows.

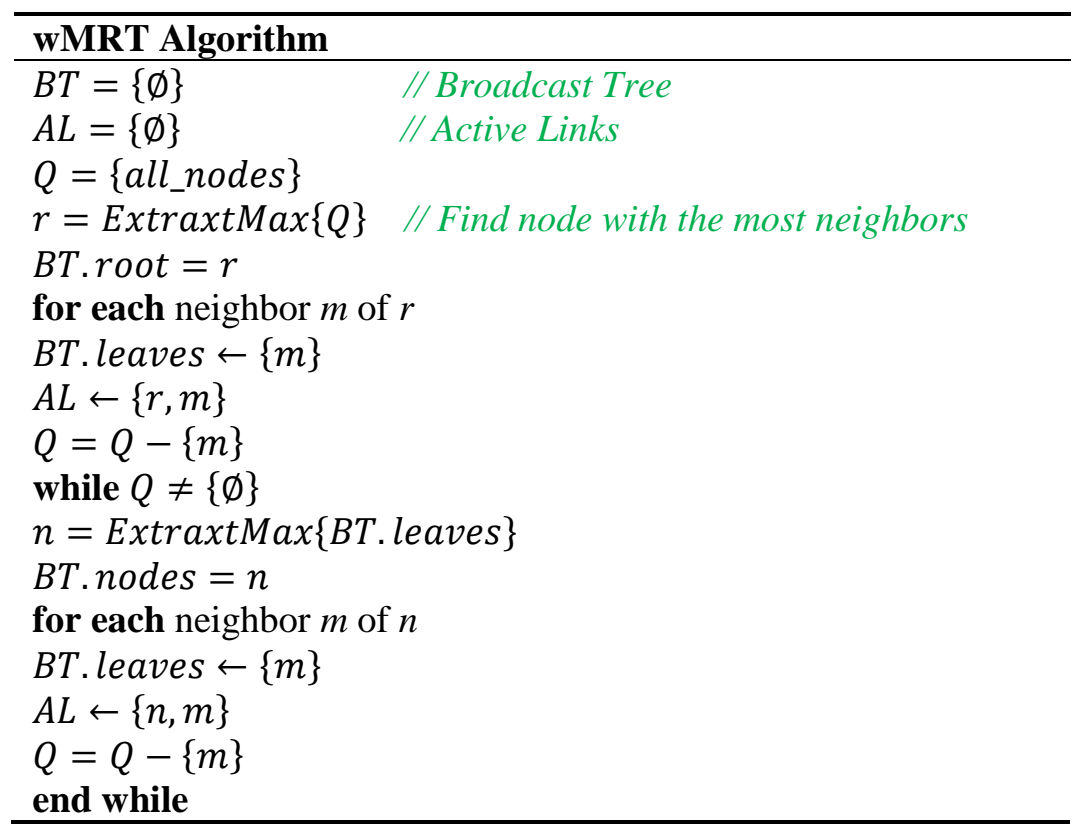

3.2.2. Broadcasting Operations: Under wMRT, when a node sends a broadcast message, it only sends the broadcast message over its active-broadcast ports. When active- 
broadcast nodes receive the broadcast message, they only send it over their activebroadcast ports, and not all their ports (as in MRT) except for the port that receives the broadcast message. Passive-broadcast nodes will not retransmit the received message. Because all passive-broadcast nodes are connected to active-broadcast nodes via activebroadcast links, the broadcast message is delivered through the network.

For the sample network with the broadcast tree shown in Figure 5, when node 1 sends a broadcast message, it only sends the message over its active-broadcast port. Activebroadcast node 7 receives and sends the message only to its active-broadcast ports, except for the port connecting to node 1 . Active-broadcast node 13 receives and sends the message only to its active-broadcast ports, except for the port connecting to node 7 . Similarly, the other active-broadcast nodes $(9,17$, and 19) receive and send the message only to their active-broadcast ports, except for the ports connecting to node 13 . In that case, passive-broadcast node 8 receives only one copy of the broadcast messages that are retransmitted by active-broadcast node 7 . Similarly, the other passive-broadcast nodes receive only one copy of the broadcast message. In other words, the wMRT approach completely removes broadcast duplication in the wired network.

\subsection{Maintaining and Updating the Broadcast Tree}

When any link state change occurs in a wired network, the link state routing protocol exchanges the link information messages and updates the link state table of the network.

Once the link table is updated, wMRT recalculates the broadcast tree of the network based on the latest updated link table and updates the broadcast roles for the nodes and ports of the nodes in the network.

\section{Performance Analysis}

This section describes an analysis of the broadcast traffic performance of the wMRT technique compared to the simple flooding and MRT techniques.

In this paper, the measures of broadcast traffic and broadcast duplication are used in order to analyze and evaluate the broadcast traffic performance of the broadcasting techniques.

- Broadcast traffic: Broadcast traffic in a network is defined as the total number of broadcast messages that are delivered in links and received by nodes in the network.

- Broadcast duplications: Broadcast duplications are the total number of broadcast messages that are received and discarded by all nodes due to duplication.

When a node sends a broadcast message to an $n$-node network, all of the other nodes expect to receive a copy of the broadcast message. In other words, $n-1$ copies of the broadcast message are expected to be received at $n-1$ nodes, except for the sender node. After receiving the first copy of the broadcast message, if the nodes continue to receive more copies of the message, the copies are considered to be broadcast duplications. Therefore, broadcast duplications are $n-1$ less than broadcast traffic.

The sample wired network consisting of 25 nodes as shown in Figure 1 is used to analyze and evaluate the broadcast traffic performance of the broadcasting techniques.

\subsection{Under Simple Flooding}

For the simple flooding technique, when a node sends a broadcast message, all of the nodes (except the sending node) retransmit the message when it is first received. Figure 6 shows a message being broadcast in the sample network under simple flooding. 


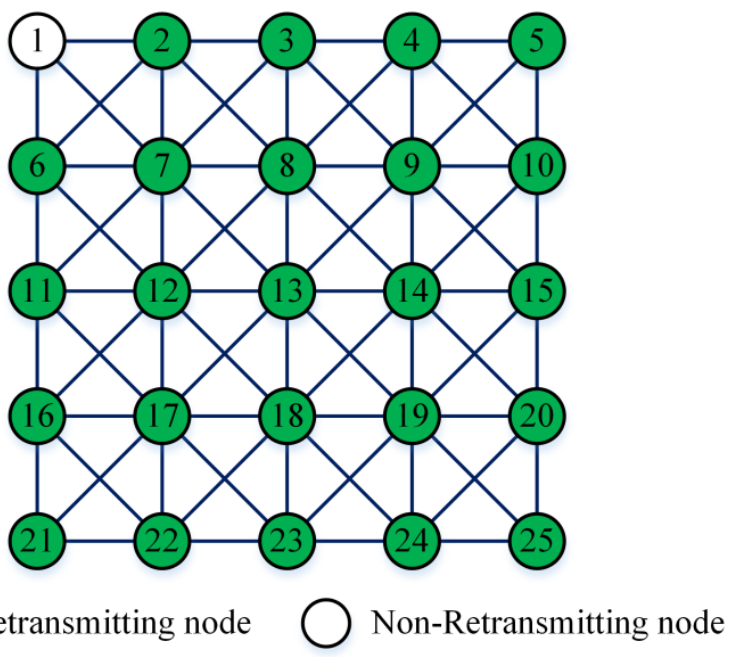

Figure 6. Broadcasting a Message under Simple Flooding

Under simple flooding, broadcast traffic when a node sends a broadcast message, denoted by $t f_{\text {sim }}^{1}$, is calculated as follows:

$$
t f_{\text {sim }}^{1}=N_{\text {link }}^{S}+\sum_{i \in S_{\text {sim }}^{r t x}}\left(N_{\text {link }}^{i}-1\right)
$$

where $N_{\text {link }}^{S}$ is the number of links of the sender node, $S_{\text {sim }}^{r t x}$ is a set of retransmitting nodes, and $N_{\text {link }}^{i}$ is the number of links of the $i^{\text {th }}$ node.

The sum of the links of all retransmitting nodes is determined as follows:

$$
\sum_{i \in S_{\text {sim }}^{r t x}} N_{\text {link }}^{i}=2 N_{\text {link }}-N_{\text {link }}^{S}
$$

where $\mathrm{N}_{\text {link }}$ is the total number of links in the network.

Equation (1) can be rewritten as follows:

$$
t f_{\text {sim }}^{1}=2 N_{\text {link }}-N_{\text {sim }}^{r t x}=2 N_{\text {link }}-\left(N_{\text {node }}-1\right)
$$

where $N_{\text {node }}$ is the total number of nodes in the network, and $N_{\text {sim }}^{r t x}=N_{\text {node }}-1$ is the number of retransmitting nodes.

Broadcast duplications when a node sends a broadcast message, denoted by $\mathrm{dup}_{\text {sim }}^{1}$, are determined as follows:

$$
\mathrm{dup}_{\text {sim }}^{1}=t f_{\text {sim }}^{1}-\left(N_{\text {node }}-1\right)
$$

By replacing (3) with (4), the broadcast duplications can be calculated as follows:

$$
d u p_{\text {sim }}^{1}=2\left(N_{\text {link }}-N_{\text {node }}+1\right)
$$

In general, broadcast duplications when a node sends $N$ broadcast messages, denoted by $d u p_{\text {sim }}$, are calculated as follows:

$$
\operatorname{dup}_{\text {sim }}=2 N\left(N_{\text {link }}-N_{\text {node }}+1\right)
$$

For the sample network in Figure 1, broadcast duplications are calculated as follows:

$$
d u p_{\text {sim }}=2 N(72-25+1)=96 N
$$

\subsection{Under MRT}

Under MRT flooding, when a node sends a broadcast message, it floods the message to all of its ports. When active-broadcast nodes receive the message, they flood the message to all of their ports, except for the received port. When passive-broadcast nodes receive the message, they do not retransmit it. Figure 4 shows a message being broadcast in the sample network under MRT. We consider the following scenarios:

- Broadcast messages are sent by a passive-broadcast node.

- Broadcast messages are sent by an active-broadcast node. 
4.2.1. Sent by a Passive-broadcast Node: Broadcast traffic when a passive-broadcast node sends a broadcast message, denoted by $t f_{M R T}^{1}$, is calculated as follows:

$$
t f_{M R T}^{1}=N_{\text {link }}^{S}+\sum_{i \in S_{M R T}^{a c t}}\left(N_{\text {link }}^{i}-1\right)
$$

where $N_{\text {link }}^{S}$ is the number of links of the sender node and $S_{M R T}^{a c t}$ is a set of activebroadcast nodes.

When a passive-broadcast node sends a broadcast message, broadcast duplications, denoted by $d u p_{M R T}^{1}$, are determined as follows:

$$
\operatorname{dup}_{M R T}^{1}=N_{\text {link }}^{S}+\sum_{i \in S_{M R T}^{a c t}}\left(N_{\text {link }}^{i}-1\right)-\left(N_{\text {node }}-1\right)
$$

In general, broadcast duplications when a passive-broadcast node sends $N$ broadcast messages, denoted by $\operatorname{dup}_{M R T}$, are calculated as follows:

$$
\operatorname{dup}_{M R T}=N\left(N_{\text {link }}^{S}+\sum_{i \in S_{M R T}^{a c t}}\left(N_{\text {link }}^{i}-1\right)-\left(N_{\text {node }}-1\right)\right)
$$

For the sample network in Figure 1, when node 1 sends $N$ broadcast messages, broadcast duplications are calculated as follows:

$$
\operatorname{dup}_{M R T}=N(3+5 \times 7-25+1)=14 N
$$

4.2.2. Sent by an Active-broadcast Node: When an active-broadcast node sends a broadcast message, broadcast traffic, denoted by $t f_{M R T}^{1}$, is calculated as follows:

$$
t f_{M R T}^{1}=N_{\text {link }}^{S}+\sum_{i \in S_{M R T}^{a c t, I S}}\left(N_{\text {link }}^{i}-1\right)
$$

where $S_{M R T}^{a c t, I s}$ is a set of active-broadcast nodes (except for the sending node).

$$
\sum_{i \in S_{M R T}^{a c t}} N_{\text {link }}^{i}=N_{\text {link }}^{S}+\sum_{i \in S_{M R T}^{a c t ! s}} N_{\text {link }}^{i}
$$

Equation (10) can be rewritten as follows:

$$
t f_{M R T}^{1}=1+\sum_{i \in S_{M R T}^{a c t}}\left(N_{\text {link }}^{i}-1\right)
$$

When an active-broadcast node sends a broadcast message, broadcast duplications, denoted by $d u p_{M R T}^{1}$, are determined as follows:

$$
\operatorname{dup}_{M R T}^{1}=\sum_{i \in S_{M R T}^{a c t}}\left(N_{\text {link }}^{i}-1\right)-\left(N_{\text {node }}-2\right)
$$

In general, broadcast duplications when an active-broadcast node sends $N$ broadcast messages, denoted by $\operatorname{dup}_{M R T}$, are calculated as follows:

$$
\operatorname{dup}_{M R T}=N\left(\sum_{i \in S_{M R T}^{a c t}}\left(N_{\text {link }}^{i}-1\right)-\left(N_{\text {node }}-2\right)\right)
$$

For the sample network in Figure 1, when node 7 sends $N$ broadcast messages, broadcast duplications are calculated as follows:

\subsection{Under wMRT}

$$
\operatorname{dup}_{M R T}=N(5 \times 7-25+2)=12 N
$$

Under wMRT, when a node sends a broadcast message, it only sends the message over its active-broadcast ports. When active-broadcast nodes receive the message, they only send the message to their active-broadcast ports, except for the receiving port. When 
passive-broadcast nodes receive the message, they discard it. Figure 5 shows a message being broadcast in the sample network under wMRT. We consider the following scenarios:

- Broadcast messages are sent by a passive-broadcast node (node 1).

- Broadcast messages are sent by an active-broadcast node (node 7).

4.3.1. Sent by a Passive-broadcast Node: Broadcast traffic when a passive-broadcast node sends a broadcast message, denoted by $t f_{W M R T}^{1}$, is calculated as follows:

$$
t f_{w M R T}^{1}=N_{a p}^{S}+\sum_{i \in S_{w M R T}^{a c t}}\left(N_{a p}^{i}-1\right)
$$

where $N_{a p}^{S}$ is the number of active-broadcast ports of the sender node $\left(N_{a p}^{S}=1\right.$ for passive-broadcast nodes), $S_{w M R T}^{a c t}$ is a set of active-broadcast nodes, and $\mathrm{N}_{\text {ap }}^{\mathrm{i}}$ is the number of active-broadcast ports of the $i^{\text {th }}$ node.

Broadcast duplications when a passive-broadcast node sends a broadcast message, denoted by $\operatorname{dup}_{w M R T}^{1}$, are determined as follows:

For the broadcast tree,

$$
d u p_{w M R T}^{1}=\sum_{i \in S_{w M R T}^{a c t}}\left(N_{a p}^{i}-1\right)-\left(N_{\text {node }}-2\right)
$$

$$
\sum_{i \in S_{w M R T}^{a c t}}\left(N_{a p}^{i}-1\right)=N_{\text {link }}^{a c t}-1=N_{\text {node }}-2
$$

where $\mathrm{N}_{\text {link }}^{\text {act }}$ is the number of active-broadcast links $\left(\mathrm{N}_{\text {link }}^{\text {act }}=\mathrm{N}_{\text {node }}-1\right)$.

Therefore, broadcast duplications in (16) can be determined as:

$$
d u p_{w M R T}^{1}=N_{\text {node }}-2-\left(N_{\text {node }}-2\right)=0
$$

4.3.2. Sent by an Active-broadcast Node: Broadcast traffic when an active-broadcast node sends a broadcast message, denoted by $\mathrm{tf}_{\mathrm{wMRT}}^{1}$, is calculated as follows:

$$
t f_{w M R T}^{1}=N_{a p}^{S}+\sum_{i \in S_{w M R T}^{a c t, ! S}}\left(N_{a p}^{i}-1\right)
$$

where $S_{\text {wMRT }}^{\text {act,IS }}$ is a set of active-broadcast nodes (except for the sender node).

$$
\sum_{i \in S_{M R T}^{a c t}} N_{a p}^{i}=N_{a p}^{i}+\sum_{i \in S_{w M R T}^{a c t, ! s}} N_{a p}^{i}
$$

Equation (19) can be rewritten as follows:

$$
t f_{w M R T}^{1}=1+\sum_{i \in S_{w M R T}^{a c t}}\left(N_{a p}^{i}-1\right)=N_{\text {node }}-1
$$

Broadcast duplications when an active-broadcast node sends a broadcast message, denoted by dup $\mathrm{w}_{\mathrm{wMRT}}^{1}$, are determined as follows:

$$
d u p_{w M R T}^{1}=t f_{w M R T}^{1}-\left(N_{\text {node }}-1\right)=0
$$

Therefore, when an active-broadcast or passive-broadcast node sends broadcast messages, there is no broadcast duplication in the wired network under the wMRT approach.

\section{Simulations}

In order to validate the analytical results derived in Section 4, various simulations were carried out using a network simulation tool, OMNeT++v4.6 [24].

\subsection{Simulation Model}

The objective of the simulations is to validate the analytical results and compare the broadcast traffic performance of the proposed wMRT technique with that of the simple flooding and the original MRT technique in a wired network. 
Networks used in the simulations were wired networks with network size of $m \times m$ nodes, as shown in Figure 7.

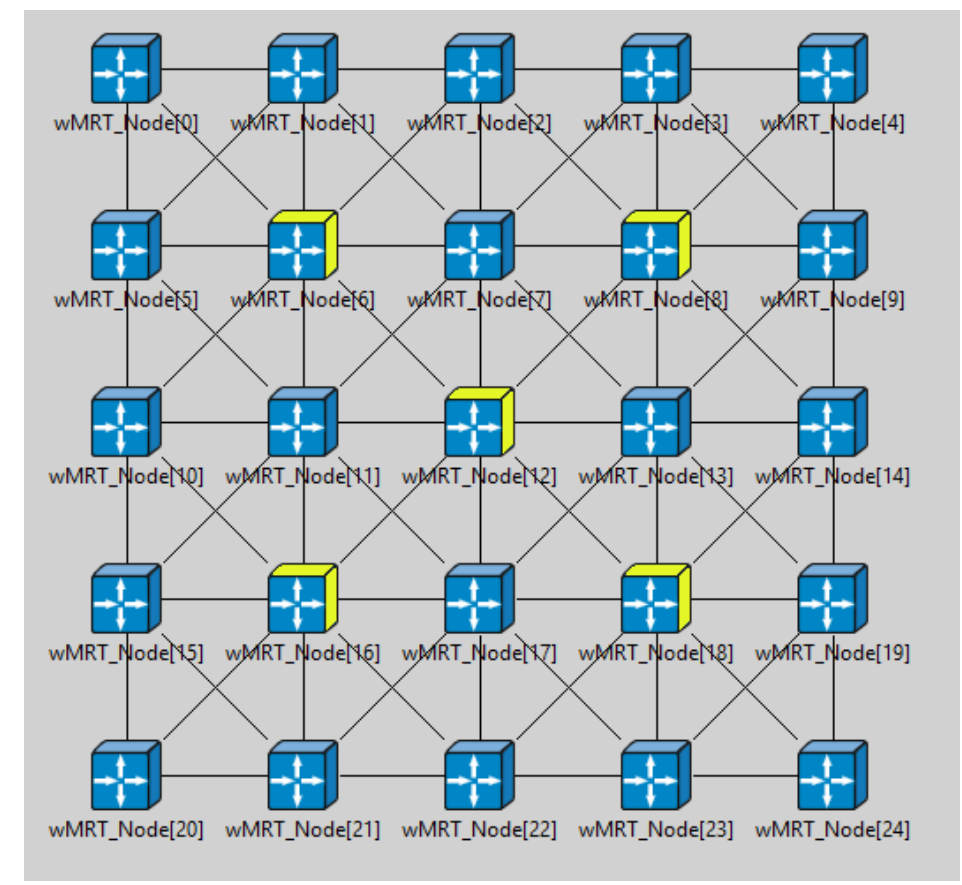

Figure 7. A Simulation Network with Network Size of $5 \times 5$ Nodes

Because both the MRT and wMRT techniques deliver broadcast messages based on the broadcast tree of a network that is constructed based on the network's link information, it is necessary to build a link table that describes a map of the connectivity to the network.

In the simulations, the link tables of simulation networks were built by using the BERbased routing protocol (BRP) [25]. The BRP's nodes first discover their neighboring nodes, then exchange their links' information, and finally build the link table based on the received link information.

The process of building a network link table in the BRP model is briefly described as follows.

- Discovering neighbors: First, every node discovers its available neighbors. A Hello message is used to discover neighbors. Each node receives the Hello messages from its neighbors and then builds its own neighbor table.

- Exchanging BER information: To build the link table, nodes first send their links' information to each other. Each node advertises its links' information by broadcasting a Link-metric message.

- Building the link table: Based on the received Link-metric messages, each node builds its own link table. For a connected network, all nodes in the network have the same link table.

Based on the link table built by the BRP, the MRT and wMRT algorithms create the broadcast tree of the network.

\subsection{Simulation Description}

Two simulations were conducted to validate and evaluate the broadcast traffic performance of the proposed wMRT technique.

5.2.1. Simulation 1: The first simulation was performed to validate the broadcast traffic performance analysis in Section 4. We considered the sample wired network with 25 
nodes, as shown in Figure 1. The sender node sent $\mathrm{N}$ broadcast messages to the network $(\mathrm{N}=10,20, \ldots, 100)$. The broadcast traffic and broadcast duplication under the flooding techniques were recorded for comparison with the traffic performance analyzed in Section 4.

We considered the following cases:

- Case 1: Broadcast messages were sent by a passive-broadcast node.

- Case 2: Broadcast messages were sent by an active-broadcast node.

5.2.2. Simulation 2: The second simulation was implemented in various simulated networks with different numbers of nodes. Each network had $m \times m$ nodes $(m=3,4, \ldots$, 10). The simulation was performed under the simple flooding, MRT, and wMRT approaches. Sender node 1 sent 50 broadcast messages to the networks. The simulation results were recorded and used to evaluate and compare the broadcast traffic performance of the flooding techniques.

\subsection{Simulation Results}

5.3.1. Simulation 1: The results of the simulation 1 are shown in Table 1 and Table 2 .

Table 1. Simulation Results of Case 1 in Simulation 1

\begin{tabular}{c||c|c|c|c|c|c}
\hline \multicolumn{1}{c||}{\multirow{1}{*}{$\begin{array}{c}\text { Sent } \\
\text { messages }\end{array}$}} & \multicolumn{3}{c|}{ Broadcast traffic } & \multicolumn{3}{c}{ Broadcast duplications } \\
\cline { 2 - 7 } & SP & MRT & wMRT & SP & MRT & wMRT \\
\hline 10 & 1,200 & 380 & 240 & 960 & 140 & 0 \\
20 & 2,400 & 760 & 480 & 1,920 & 280 & 0 \\
30 & 3,600 & 1,140 & 720 & 2,880 & 420 & 0 \\
40 & 4,800 & 1,520 & 960 & 3,840 & 560 & 0 \\
50 & 6,000 & 1,900 & 1,200 & 4,800 & 700 & 0 \\
60 & 7,200 & 2,280 & 1,440 & 5,760 & 840 & 0 \\
70 & 8,400 & 2,660 & 1,680 & 6,720 & 980 & 0 \\
80 & 9,600 & 3,040 & 1,920 & 7,680 & 1,120 & 0 \\
90 & 10,800 & 3,420 & 2,160 & 8,640 & 1,260 & 0 \\
100 & 12,000 & 3,800 & 2,400 & 9,600 & 1,400 & 0 \\
\hline
\end{tabular}

Table 2. Simulation Results of Case 2 in Simulation 1

\begin{tabular}{c||c|c|c|c|c|c}
\hline \multicolumn{1}{c||}{ Sent } & \multicolumn{3}{c|}{ Broadcast traffic } & \multicolumn{3}{c}{ Broadcast duplications } \\
\cline { 2 - 6 } messages & SP & MRT & wMRT & SP & MRT & wMRT \\
\hline 10 & 1,200 & 360 & 240 & 960 & 120 & 0 \\
20 & 2,400 & 720 & 480 & 1,920 & 240 & 0 \\
30 & 3,600 & 1080 & 720 & 2,880 & 360 & 0 \\
40 & 4,800 & 1440 & 960 & 3,840 & 480 & 0 \\
50 & 6,000 & 1800 & 1,200 & 4,800 & 600 & 0 \\
60 & 7,200 & 2160 & 1,440 & 5,760 & 720 & 0 \\
70 & 8,400 & 2520 & 1,680 & 6,720 & 840 & 0 \\
80 & 9,600 & 2880 & 1,920 & 7,680 & 960 & 0 \\
90 & 10,800 & 3240 & 2,160 & 8,640 & 1080 & 0 \\
100 & 12,000 & 3600 & 2,400 & 9,600 & 1200 & 0 \\
\hline
\end{tabular}

Figures $8 \mathrm{a}$ and $8 \mathrm{~b}$ show comparisons of broadcast traffic and broadcast duplication, respectively, for the simple flooding, MRT, and wMRT approaches in Case 1. 


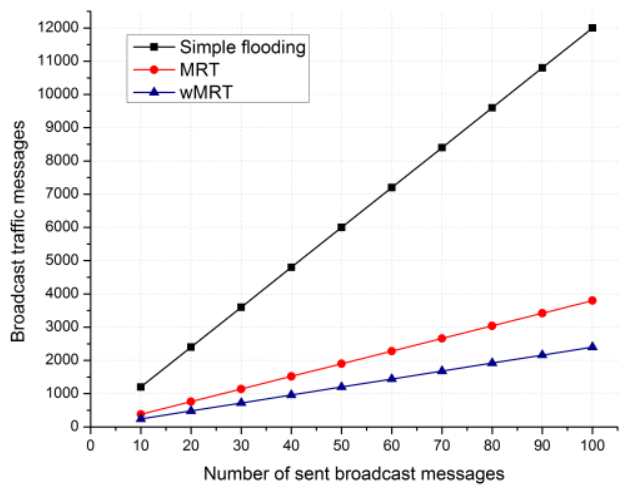

(a)

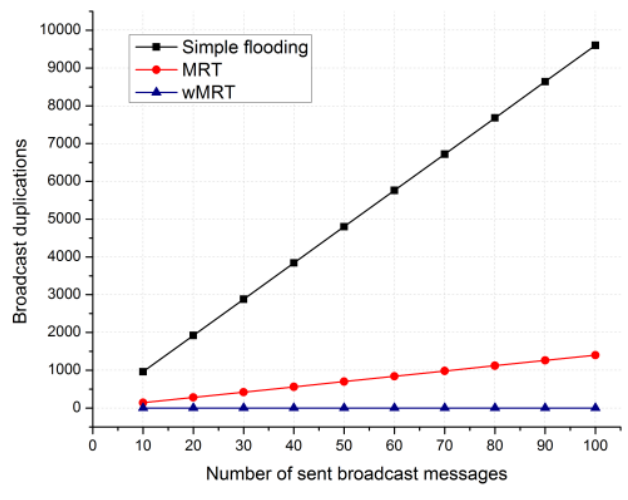

(b)

\section{Figure 8. Comparisons of (a) Broadcast Traffic and (b) Broadcast Duplications in Simulation 1's Case 1}

Figures 9a and 9b show comparisons of broadcast traffic and broadcast duplication, respectively, for these flooding techniques in Case 2.

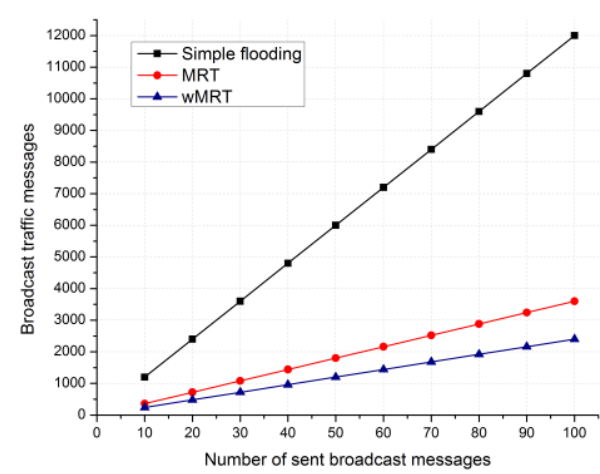

(a)

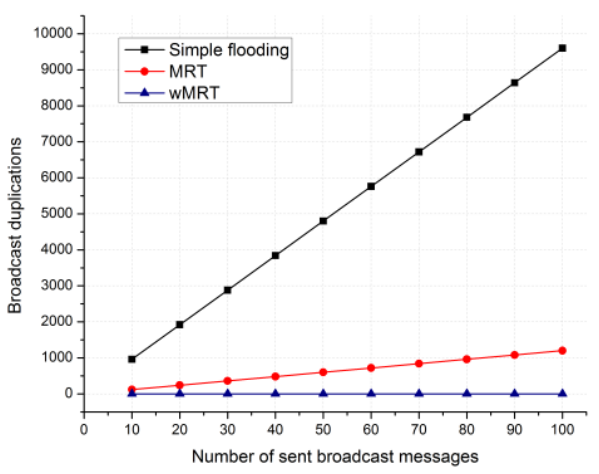

(b)

\section{Figure 9. Comparisons of (a) Broadcast Traffic and (b) Broadcast} Duplications in Simulation 1's Case 2

The simulation results showed that there was no broadcast duplication in the sample network under the wMRT. In other words, the wMRT completely removed broadcast duplication in wired networks. Therefore, the wMRT significantly reduced broadcast traffic compared with simple flooding and original MRT. For our sample network, the wMRT approach reduced broadcast traffic by $33 \%$ (in Case 2) to $37 \%$ (in Case 1) compared with MRT, and by about $80 \%$ compared with simple flooding.

5.3.2. Simulation 2: The recorded broadcast traffic and duplications of Simulation 2 are shown in Table 3. Figure 10a shows a comparison of broadcast traffic, and Figure 10b shows a comparison of broadcast duplication for the simple flooding, MRT, and wMRT techniques. As with Simulation 1's results, the simulation results also show that wMRT completely removed broadcast duplication in the sample wired networks. For our sample networks, wMRT reduced broadcast traffic by $20 \%$ to $45 \%$ compared with MRT, and by $75 \%$ to $83 \%$ compared with simple flooding. 
Table 3. Simulation Results of Case 2

\begin{tabular}{c||c|c|c|c|c|c}
\hline \multicolumn{1}{c||}{$\begin{array}{c}\text { Network } \\
\text { size }\end{array}$} & \multicolumn{3}{c|}{ Broadcast traffic } & \multicolumn{3}{c}{ Broadcast duplications } \\
\cline { 2 - 7 } & SP & MRT & wMRT & SP & MRT & wMRT \\
\hline $3 \times 3$ & 1,600 & 500 & 400 & 1,200 & 100 & 0 \\
$4 \times 4$ & 3,450 & 1,250 & 750 & 2,700 & 500 & 0 \\
$5 \times 5$ & 6,000 & 1,900 & 1,200 & 4,800 & 700 & 0 \\
$6 \times 6$ & 9,250 & 2,950 & 1,750 & 7,500 & 1,200 & 0 \\
$7 \times 7$ & 13,200 & 4,100 & 2,400 & 10,800 & 1,700 & 0 \\
$8 \times 8$ & 17,850 & 5,750 & 3,150 & 14,700 & 2,600 & 0 \\
$9 \times 9$ & 23,200 & 7,200 & 4,000 & 19,200 & 3,200 & 0 \\
$10 \times 10$ & 29,250 & 8,650 & 4,950 & 24,300 & 3,700 & 0 \\
\hline
\end{tabular}

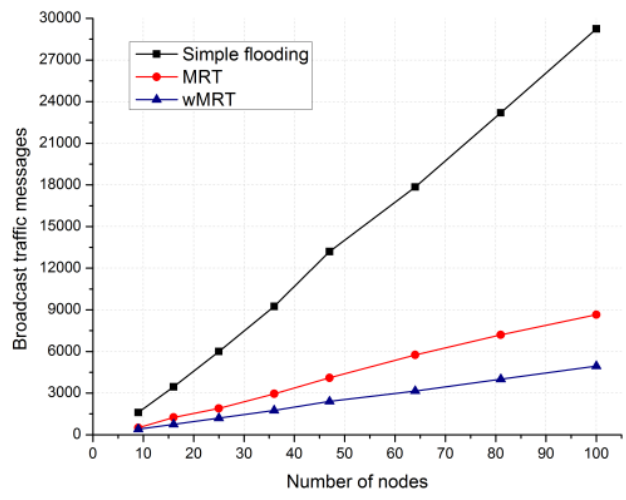

(a)

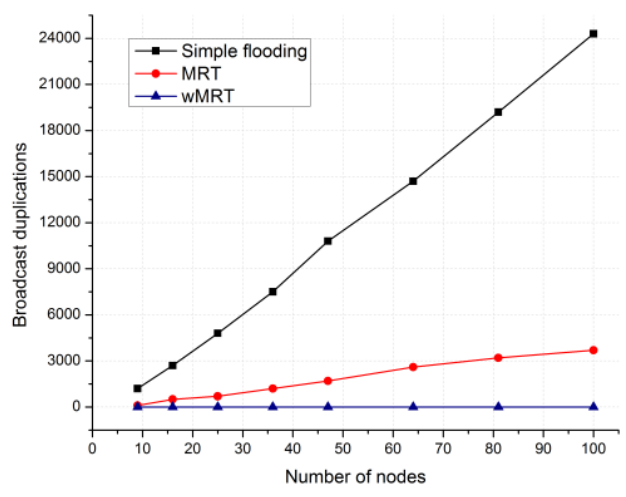

(b)

Figure 10. Comparisons of (a) Broadcast Traffic and (b) Broadcast Duplications in Simulation 2

\subsection{Discussions}

The simulation results demonstrate that the broadcast traffic performance of the proposed wMRT is much better than that of the simple flooding and original MRT approaches. As shown in Figures 8b, 9b, and 10b, while the number of broadcast duplications is too high under simple flooding and low under MRT, the number of broadcast duplications under wMRT is reduced to zero. In other words, the wMRT approach completely removes broadcast duplication from the network.

As a result of the minimization of both retransmitting nodes and retransmitting ports, MRT completely removes broadcast message duplication, and thus significantly reduces broadcast traffic messages compared to simple flooding and MRT, as shown in Figure 8a, 9a, and 10a. Numerically, for our sample networks, wMRT reduced broadcast traffic by $20 \%$ to $45 \%$ compared with MRT, and by $75 \%$ to $83 \%$ compared with simple flooding.

\section{Conclusions}

In this paper, we developed a variant of the MRT approach for broadcasting in wired networks, called wMRT. Unlike MRT, which allows active-broadcast nodes to flood a received broadcast message to all of their ports, the proposed wMRT allows the activebroadcast nodes to only retransmit the received broadcast message over their activebroadcast ports, instead of all ports. By minimizing both the number of active-broadcast nodes and the number of active-broadcast ports, the wMRT approach significantly reduces the number of broadcast traffic messages and completely prevents broadcast messages from being duplicated in wired networks, thus saving a significant amount of network bandwidth, as well as improving network traffic performance. The proposed 
wMRT is a broadcasting technique that is highly suitable for wired networks working with link state routing protocols.

\section{Acknowledgments}

This research was supported by Basic Science Research Program through the National Research Foundation of Korea (NRF) funded by the Ministry of Education (NRF2015R1D1A1A02059506).

\section{References}

[1] W. Woon and K. L. Yeung, "A New Phase for Screening Redundant Broadcast Nodes in SourceIndependent Broadcasting Protocols", Proceedings of 2011 IEEE International Conference on Communications (ICC), Kyoto, Japan, (2011).

[2] C. E. Perkins and E. M. Royer, "Ad Hoc On-demand Distance Vector Routing", Proceedings of the 2nd Annual IEEE International Workshop on Mobile Computing Systems and Applications, (1999).

[3] D. B. Johnson and D. A. Maltz, "Dynamic Source Routing in Ad Hoc Wireless Networks", The Kluwer International Series in Engineering and Computer Science, (1996), pp. 153-181.

[4] RFC 2328: OSPF Version 2. Available online at https://tools.ietf.org/html/rfc2328.

[5] RFC 1142: OSI IS-IS Intra-domain Routing Protocol. Available online at https://tools.ietf.org/html/rfc1142.

[6] A. Hu, "Video-on-Demand Broadcasting Protocols: A Comprehensive Study", Proceedings of the Twentieth Annual Joint Conference of the IEEE Computer and Communications Societies (INFOCOM), (2001), pp. 508-517.

[7] J. W. Lin, "Broadcast Scheduling for a P2P Spanning Tree", Proceedings of 2008 IEEE International Conference on Communications (ICC), (2008), pp. 5614-5618.

[8] N. Karthikeyan, V. Palanisamy, and K. Duraiswamy, "A Review of Broadcasting Methods for Mobile Ad Hoc Network", International Journal of Advanced Computer Engineering, (2009).

[9] B. Williams and T. Camp, "Comparison of Broadcasting Techniques for Mobile Ad Hoc Networks", Proceedings of the 3rd ACM international symposium on Mobile ad hoc networking \& computing, (2002), pp. 194-205.

[10] P. Nand and S. C. Sharma, "Comparative Analysis of Broadcasting Techniques for Routing Protocols", Proceedings of International Conference on Devices and Communications (ICDeCom), (2011), pp. 1-5.

[11] I. M. Mkwawa and D. D. Kouvatsos, "Broadcasting Methods in MANETS: An Overview," Network Performance Engineering, Springer, Heidelberg, (2011), pp. 767-783.

[12] N. X. Tien and J. M. Rhee, "Minimizing Re-Transmissions (MRT) for Broadcast Traffic in Ad Hoc Wireless Networks," IEICE Transactions on Communications, vol. E99-B, 01, (2016), pp. 257-268.

[13] N. X. Tien and J. M. Rhee, "A Novel Variant of the Minimizing Re-Transmissions Technique for Wired Networks", Asia-pacific Proceedings of Applied Science and Engineering for Better Human Life, Jeju, Korea, vol. 4, (2016), pp. 102-106.

[14] S. Viswanathan and T. Imielinski, "Pyramid Broadcasting for video on demand service", Proceedings of IEEE Multimedia Computing and Networking Conference, vol. 2417, (1995), pp. 66-77.

[15] C. C. Aggarwal, J. L. Wolf and P. S. Yu, "A permutation-based pyramid broadcasting scheme for videoon-demand systems", Proceedings of the IEEE International Conference on Multimedia Computing and Systems, (1996), pp. 118-126.

[16] L. Juhn and L. Tseng, "Harmonic broadcasting for video-on-demand service", IEEE Transactions on Broadcasting, vol. 43, no. 3, (1997), pp. 268-271.

[17] J. F. Paris, S. W. Carter and D. D. E. Long, "Efficient broadcasting protocols for video on demand", The 6th International Symposium on Modeling, Analysis and Simulation of Computer and Telecommunication Systems (MASCOTS), (1998), pp. 127-132.

[18] J. F. Paris, S. W. Carter and D. D. E. Long, "A low bandwidth broadcasting protocol for video on demand", Proceedings of IEEE International Conference on Computer Communications and Networks, (1998), pp. 690-697.

[19] J. F. Paris, S. W. Carter and D. D. E. Long, "A hybrid broadcasting protocol for video on demand", Proceedings of 1999 Multimedia Computing and Networking Conference (MMCN'99), (1999), pp. 317326

[20] J. F. Paris, "A simple low-bandwidth broadcasting protocol for video-on-demand", Proceedings of 8th International Conference on Computer Communications and Networks (IC3N'99), (1999), pp. 118-123

[21] S. El-Ansary, L. O. Alima, P. Brand and S. Haridi, "Effcient Broadcast in Structured P2P Networks," International Workshop on Peer-to-Peer Systems, (2003).

[22] A. Ghodsi, L. O. Alima, S. El-Ansary, P. Brand and S. Haridi, "Self-Correcting Broadcast in Distributed Hash Tables", Proceedings of International Conference, Parallel and Distributed Computing and Systems, (2003). 
[23] J. Li, K. Sollins and D. Y. Lim, "Implementing Aggregation and Broadcast over Distributed Hash Tables", ACM SIGCOMM Computer Communication Review, vol. 35, (2005).

[24] OMNeT++ v.4.6 Simulator. Available online: http://www.omnetpp.org/.

[25] N. X. Tien and J. M. Rhee, "The BRP: A Novel BER-Based Routing Protocol for Ad Hoc Wireless Networks," INFORMATION, vol. 19, no. 6(A), (2016), pp. 1893-1905.

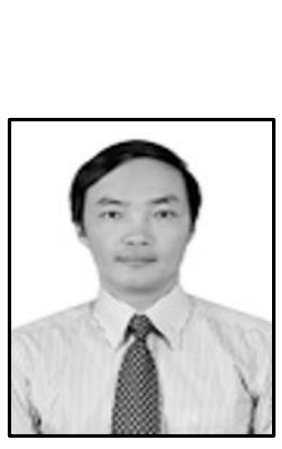

\section{Authors}

Nguyen Xuan Tien received his B.S. degree in Electronics \& Telecommunications Engineering and M.S. degree in Information Technology from Danang University of Technology in 1997 and 2004, respectively. After graduation, he joined VNPT and Beeline VN in 1997 and 2009, respectively. He served as Deputy Head of Technical Department at VNPT and Head of O\&M Department at Beeline VN. He is currently a $\mathrm{PhD}$ candidate in the Information and Communication Engineering Department at Myongji University, South Korea. His current research interests include fault-tolerant networks, traffic reduction algorithms, and ad-hoc wireless networks.

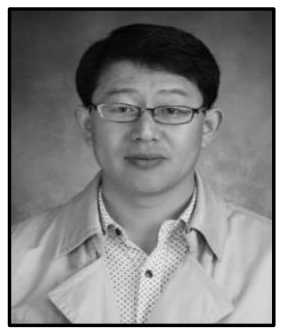

Semog Kim received $\mathrm{PhD}$ in Communications Engineering from Myongji Univ. in 2013. He joined Neodigital, distributer of Motorola Broadband technical part as a HFC network Engineer from 1998 to 2010, and RingNet, distributer of Cisco Networks as a HFC engineer until 2012. Currently, work Myongji University as invited Professor and interests are in fault-tolerant networks, smart grid communications, and HFC network.

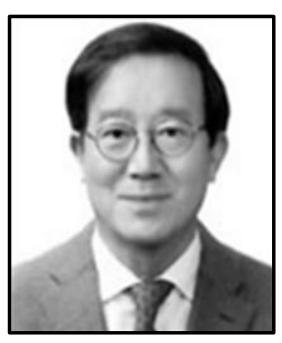

Jong Myung Rhee received his $\mathrm{PhD}$ from North Carolina State University, USA, in 1987. After 20 years at the Agency for Defense Development in Korea, where he made noteworthy contributions to C4I and military satellite communications, he joined DACOM and Hanaro Telecom in 1997 and 1999, respectively. At Hanaro Telecom, which was the second largest local carrier in Korea, he served as chief technology officer (CTO), with a senior executive vicepresident position. His main duty at Hanaro Telecom was a combination of management and new technology development for high-speed Internet, VoIP, and IPTV. In 2006 he joined Myongji University and is currently a full professor in the Information and Communications Engineering Department. His recent research interests are centered on military communications and smart grid, including ad-hoc and fault-tolerant networks. He is a member of IEEE and IEICE. 
International Journal of Future Generation Communication and Networking Vol. 9, No.10, (2016) 